\title{
If a Pig Coughs in Mexico the Whole World Should Hear It
}

\section{By Chelsea Himsworth}

We should not be breathing a sigh of relief because the H1N1 pandemic appears to be abating. ${ }^{1}$ Rather, we should be alarmed by the specter of future, potentially more disastrous, outbreaks of zoonotic diseases (diseases transmissible from animals to humans) that 'swine flu' portends. Such consternation is warranted given that (a) over $60 \%$ of all pathogens currently known to cause disease in humans have an animal origin,,$^{2}$ (b) over $75 \%$ of emerging human pathogens are zoonotic, ${ }^{2}$ and (c) zoonoses are twice as likely to be associated with emerging disease in humans compared to nonzoonotic pathogens. ${ }^{2}$ Since emergence is precipitated by population growth, global trade and travel, urbanization, agriculture, and climate change, ${ }^{3,4}$ the threat posed by zoonotic diseases is only going to increase.

Our vulnerability to outbreaks of zoonotic diseases is aggravated by traditional anthropocentric clinical- and laboratory-based disease surveillance systems that are capable of detecting zoonotic threats only after a disease has already emerged (and potentially become established) in human populations. ${ }^{4}$ This shortcoming is illustrated by the fact that H1N1 was only identified in people two months after the initial human outbreak, ${ }^{5}$ and several years after the virus likely became entrenched in swine. ${ }^{6,7}$ Similarly, while human-focused management (e.g., vaccination and antiviral treatment) may lessen the health impact of emerging zoonoses, it does not address the underlying animal and environmental factors that drive emergence, and thus cannot decrease the potential for future emergence of events.

National zoonotic disease research, surveillance, and management efforts are further crippled by being divided along disciplinary and institutional boundaries, ${ }^{4,8}$ resulting in a 'scientifically fragmented' ${ }^{4}$ approach. This problem is compounded by a lack of international communication with regard to diseases that clearly

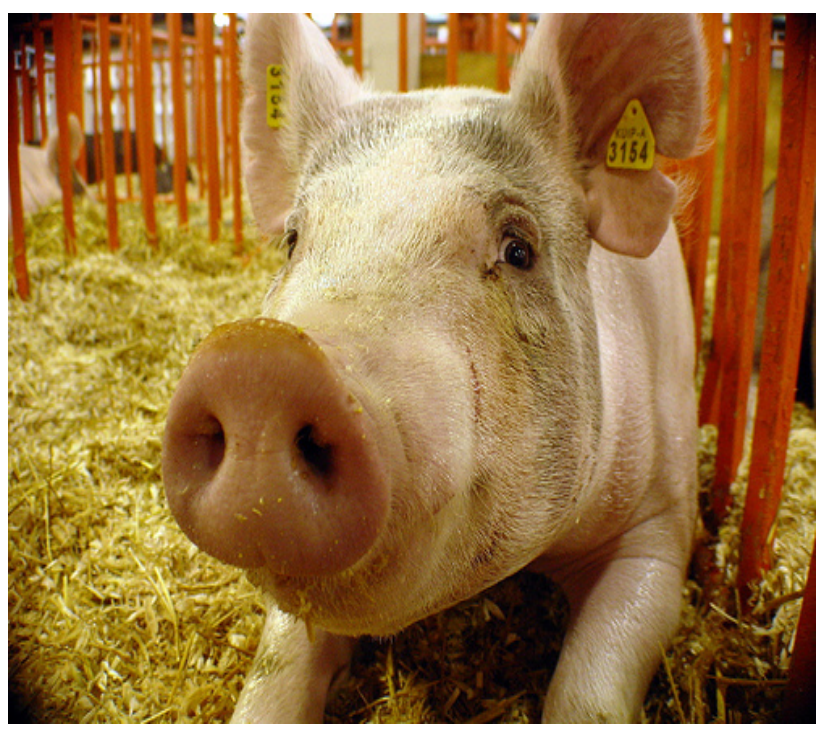

ignore political borders. ${ }^{89}$ For example, the 2009 H1N1 virus is thought to have originated from a recombination of Eurasian and North American swine viruses (likely through international trade of live pigs), ${ }^{7}$ before emerging in Mexico, and proceeding to spread to 41 countries within 4 months. ${ }^{5}$

Clearly the world is in need of an integrated, interdisciplinary, and international system for zoonotic disease surveillance and management. The closest approximation to such a system may be the Program for Monitoring Emerging Infectious Disease ${ }^{10}$ a non-governmental, freely accessible, internet-based program for reporting disease outbreaks in humans and animals. The Achilles' heel of this program is that it relies upon engaged and astute health professionals and scientists to voluntarily post information and to make use of the information posted by others, which may be the reason it is used inconsistently both within and among countries.

A more unified, scientifically-informed and policy-driven hierarchical approach to zoonotic disease is imperative. Ideally, such a system would 
consist of three levels: (1) an international body (e.g., a combination of World Health Organization and World Organisation for Animal Health) that would assume the task of designing a global surveillance and management system for emerging zoonotic diseases in humans and animals, (2) government-appointed national task forces (one per country) that would implement the system by creating networks with (3) new and/or pre-existing academic and governmental institutions throughout each country. This structure would allow fluid transfer of information between agencies, maximize proactive strategies, and foster a coordinated response to emerging zoonotic diseases. A similar approach was suggested in 2005 by Kuiken et al., who estimated that the start up-costs for such an endeavor would be approximately $\$ 4-5$ million annually for the first three years ${ }^{8}-$ a fraction of the $\$ 400$ million spent on the purchase of $\mathrm{H} 1 \mathrm{~N} 1$ vaccine in Canada alone. ${ }^{11}$

Despite the obvious need for a unified approach to zoonotic disease, such an approach has yet to be implemented. If nothing else, the H1N1 pandemic, by the very fact that it was a pandemic, should teach us that the world is not prepared to deal with emerging zoonoses. It should also teach us that we cannot afford to wait any longer before developing an integrated, interdisciplinary, and international system for zoonotic disease surveillance and management. Had such a system been in place in February, 2008, perhaps it would have "heard pigs coughing in Mexico," and been in a position to prevent, rather than merely respond to, a global pandemic.

\section{Acknowledgements}

I would like to thank Dr. F.A. Leighton and Dr. G.A. Wobeser (Department of Veterinary

Pathology, Western College of Veterinary Medicine, University of Saskatchewan) for their mentorship and intellectual guidance.

\section{References}

1. Dawood,F.S. et al. (2009) Emergence of a novel swineorigin influenza A (H1N1) virus in humans. N. Engl. J. Med. 360, 2605-2615

2. Taylor,L.H. et al. (2001) Risk factors for human disease emergence. Philos. Trans. R. Soc. Lond B Biol. Sci. 356, 983-989
3. Schrag,S.J. and Wiener,P. (1995) Emerging infectious disease: what are the relative roles of ecology and evolution? Trends in Ecology \& Evolution 10, 319-324

4. Murphy,F.A. (1998) Emerging zoonoses. Emerg. Infect. Dis. 4, 429-435

5. Neumann,G. et al. (2009) Emergence and pandemic potential of swine-origin H1N1 influenza virus. Nature 459, 931-939

6. Garten,R.J. et al. (2009) Antigenic and genetic characteristics of swine-origin 2009 A(H1N1) influenza viruses circulating in humans. Science $325,197-201$

7. Smith,G.J. et al. (2009) Origins and evolutionary genomics of the 2009 swine-origin H1N1 influenza A epidemic. Nature 459, 1122-1125

8. Kuiken,T. et al. (2005) Public health. Pathogen surveillance in animals. Science 309, 1680-1681

9. Trifonov,V. et al. (2009) Geographic dependence, surveillance, and origins of the 2009 influenza A (H1N1) virus. N. Engl. J. Med. 361, 115-119

10. (2010) About ProMED-mail. ProMED-mail http://www.promedmail.org

11. (2009) Canada to order 50.4 million H1N1 vaccine doses. CBC News http://www.cbc.ca/health/story/2009/08/06/swine-fluvaccine.html

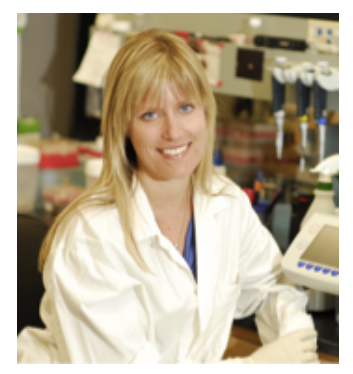

\section{Author Profile}

Chelsea Himsworth is currently a Senior Resident in the department of Veterinary Anatomic Pathology at the University of Saskatchewan. She will be starting her studies in Health Care and Epidemiology as a $\mathrm{PhD}$ candidate at the University of British Columbia School of Population and Public Health this coming September. Her research interests include ecology of disease in the wildlife, public health, and emerging zoonoses. 